\title{
Spatial memory and the performance of rats and pigeons in the radial-arm maze
}

\author{
ALAN B. BOND, ROBERT G. COOK, and MARVIN R. LAMB \\ University of California, Berkeley, California 94720
}

\begin{abstract}
The resource-distribution hypothesis states that the ability of an animal to remember the spatial location of past events is related to the typical distribution of food resources for the species. It appears to predict that Norway rats would perform better than domestic pigeons in tasks requiring spatial event memory. Pigeons, tested in an eight-arm radial maze, exhibited no more than half of the memory capacity observed in rats in the same apparatus and may not have used spatial memory at all. The results were interpreted as supporting the hypothesis.
\end{abstract}

The role of natural selection in shaping learning processes in animals has long been recognized. Studies of taste aversion and avoidance learning, for example, suggest that the ease of acquiring certain associations and their resistance to interference and extinction can be thought of as adaptations to particular features of the animal's natural environment (Hinde \& Stevenson-Hinde, 1973). In this paper, we address the question of whether the mechanisms that determine the processing and retention of spatial information are similarly susceptible to evolutionary modification. Do animal species differ in their abilities to encode and recall information about the location of particular past events, and are the differences predictable from a knowledge of the selective factors involved?

If they are to forage optimally, animals in which the primary food resources are diffusely distributed, irregularly available, and readily depleted should systematically avoid regions that have previously been exploited. Although there are several means of achieving this end without the use of memory, any ability to remember areas that were searched and exhausted during the current feeding episode should be of great selective advantage. We would therefore expect this type of resource to be commonly associated with a memory for recent foraging locations (Olton \& Schlosberg, 1978).

On the other hand, animals whose food resources are concentrated and dependable should tend to return repeatedly to the same location in successive foraging episodes. This type of resource could be exploited successfully using only a memory for locations provided by longer term associations. As a con-

We wish to thank D. A. Riley for advice, encouragement, and critical discussion throughout the course of this research. Funds for this study were provided in part by a National Institute of Mental Health Research Service Award, No. 1 T32 MH15860-01, and by NIMH Grant MH22153 and NSF Grant BNS7908839 to D. A. Riley. sequence, no memory process for recently experienced spatial locations would be selected for.

There is evidence for this hypothetical relationship between memory and the distribution of food resources. Norway rats appear to employ a memory for spatial events in laboratory preparations that are presumed to mimic food distributions typically found in the wild (Olton \& Collison, 1979; Olton, Collison, \& Werz, 1977; Olton \& Samuelson, 1976). Field studies of the foraging behavior of nectar-feeding birds suggest that they possess a similar capacity (Gill \& Wolf, 1977; Kamil, 1978). In each of these cases, the ecology of the animal is in line with the resourcedistribution hypothesis: an ability to remember recent foraging locations is associated with the exploitation of diffuse, irregular, and slowly recovering food resources. The hypothesis also requires, however, that we be able to demonstrate poor event memory in an animal that feeds primarily on dependably and abundant resources.

The domestic pigeon appears to fulfill this condition. In the wild, pigeons feed on fallen grains in agricultural areas or open grasslands, habitats that are unlikely to be depleted within a single feeding episode (Goodwin, 1967). They seldom venture to forage solitarily in unknown regions, relying instead on the presence of other birds to signal the occurrence of locally abundant resources. As a result, individual flocks develop traditional feeding sites, areas that have proved in the past to provide abundant food and safety from predation (Goodwin, 1967). Pigeons have excellant long-term memory for the locations of food sources and nest lofts, and they can be remarkably persistent in returning to such places months or even years after their last experience there, and in the absence of maintained reward (Levi, 1974). The foraging ecology of pigeons therefore contrasts sharply with that of rats and nectar-feeding birds, and we might expect that pigeons would exhibit greater difficulty in mastering a task that required spatial event memory. 
To evaluate the ability of pigeons to recall spatial events, we used a modified version of the eight-arm radial maze (Olton \& Samuelson, 1976), an apparatus in which small quantities of food are placed at each of eight separate spatial locations. Optimal performance in the maze entails a search of all eight locations without repeating prior choices. Both rats and pigeons were tested in this apparatus, and their performance was compared with one another and with the predictions of a series of memory hypotheses.

\section{METHOD}

\section{Subjects}

The subjects were 6 Silver King pigeons and 10 adult male Long-Evans rats. Prior to this experiment, the pigeons had been used in autoshaping, conditional discrimination, and diet selection experiments. The rats were experimentally naive. The birds were maintained at $75 \%$ of their free-feeding weights for the course of the experiment, while the rats were kept at $80 \%-85 \%$.

\section{Apparatus}

To accommodate the pigeons, the apparatus was about twice the size of Olton and Samuelson's (1976) original design. The central platform was $54 \mathrm{~cm}$ in diameter. Eight arms, $160 \mathrm{~cm}$ long and $22 \mathrm{~cm}$ wide, radiated out from the center at equal intervals. The maze was constructed from white-enameled wood and elevated $64 \mathrm{~cm}$ above the floor. Each arm was enclosed in a sleeve of $1.3-\mathrm{cm}$ aviary netting, approximately $34 \mathrm{~cm}$ high, to prevent the escape of the birds. The central platform was covered with a wire-mesh lid. A mesh door at the end of each arm allowed the experimenter access to a half-circular green plastic food cup (Planit), approximately $4.5 \mathrm{~cm}$ deep and $7 \mathrm{~cm}$ in radius. To improve traction and facilitate cleaning, the floor of the maze was covered with brown wrapping paper. The apparatus was placed in a large, vacant classroom that contained abundant extramaze cues, such as desks, chairs, and windows, and had uniform overhead fluorescent lighting.

\section{Procedure}

Before each session, the food cups were baited with 3-4 45-mg Noyes pellets, in the case of the rats, or 6-8 pieces of pigeon grain for the birds. Each animal was placed on the central platform in a random orientation, and the experimenter then withdrew to a distance of about $7 \mathrm{~m}$. The sequence of arms visited and the direction of the turn made when exiting each arm were recorded. The animal had to walk the entire length of an arm to determine whether a bait was present, because the lip of the cup obstructed a view of the contents at distances greater than about $20 \mathrm{~cm}$. The session was terminated when the animal had spent $30 \mathrm{~min}$ in the maze, had made 16 choices, or had consumed all eight baits. The pigeons exhibited little spontaneous exploration early in training, requiring an additional convention. If the animal had not moved for $5 \mathrm{~min}$, the experimenter slowly approached the maze and drove the animal onto the central platform. Although recording was continued after this forced movement, the results were considered to have been contaminated, and that session was not included in the analysis. The animals were tested daily for 3 weeks, of which the last $\mathbf{1 0}$ days were used in the analysis. To avoid artifacts due to the presence of the other species, the data were recorded in separate blocks. The pigeon phase of the experiment was run during August 1979. The maze was then thoroughly cleaned, and the rat phase took place the following December.

\section{RESULTS}

During the last 10 sessions, the rats required a mean of 8.76 choices to remove the food from all eight arms. On the average, 7.55 of their first 8 choices were "correct," in that they represented the first visit to that arm during the given session. This performance was significantly better than the expectation, assuming random, independent choices, of 5.25 choices $[\mathrm{t}(9)=20.2, \mathrm{p}<.001$; Olton, 1978], and consistent with those of earlier studies (Magni, Krekule, \& Bureš, 1979; Olton \& Samuelson, 1976; Olton \& Schlosberg, 1978). The pigeons made an average of 13.33 choices per session, achieving a mean of 6.28 correct choices out of the first 8 . This was also significantly better than random expectation $[t(5)=11.3, p<.001]$, but it was inferior to the performance of the rats $[t(14)=7.7, p<.001]$.

Because the experimental design entails sampling without replacement, however, the foregoing analysis is unable to provide a direct estimate of the magnitude of the memory task involved. It is not the case, for example, that the pigeons' performance implies that they remember all of the arms they chose correctly $(6.28)$ or even that they remember the number of arms in excess of the random expectation (6.28 $-5.25=1.03$ ). In addition, the number correct out of the first eight choices is clearly affected by more than just a memory for spatial events. As has been observed in several earlier studies (Olton, 1978), animals in the radial maze tend to make turns of fairly constant magnitude and direction when leaving an arm. A response bias of this sort can, of itself, yield a better than random performance, since it causes the animal to move away from arms that have already been searched. The number of correct choices is, therefore, contaminated, as a measure of spatial memory, with an unknown component attributable to the bias in the distribution of turns.

To begin to deal with these problems, we devised two novel and complementary approaches to the analysis of radial-arm maze performance. The first involved a Monte Carlo simulation of choice behavior, based solely on the empirical distribution of turn probabilities, to estimate that portion of the observed performance that could be accounted for by response bias alone. The second consisted of a simple memory model which assumes that the animal remembers some subset of its previous choices and selects randomly from among the remaining arms. By varying the size of the remembered set and comparing the predictions of the model to the empirical results, we obtained a rough estimate of the actual memory capacity required.

In both of these analyses, the probability of a correct choice as a function of choice number was used as the principal analytical measure. Curves of this measure averaged across all subjects for each species are indicated by filled triangles in Figure 1 . The expected value of this function, under the null hypothesis of independent, random choices, can be expressed as $[(n-1) / n]^{r-1}$, where $n$ is the number of arms in the maze and $r$ is the number of choices 


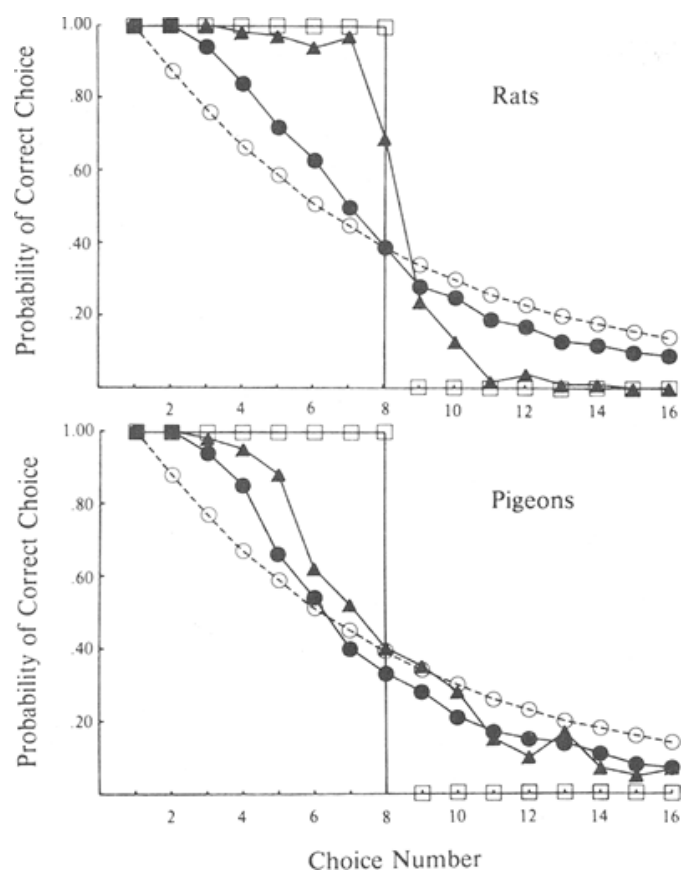

Figure 1. Probability of choosing a correct arm, as a function of choice sequence. Filled triangles indicate mean observed performance; filled circles indicate simulated performance, with choices selected randomly from the empirical probability distribution of turn magnitude. The expected value, under the assumption of random, independent cholces, is shown with a dashed line and open circles, while perfect performance is indicated by open squares.

(Parzen, 1960, p. 368). The expected probability of a correct choice thus declines as a power function of the number of choices, asymptotically approaching the $\mathrm{x}$-axis. This function is indicated by a dashed line and open circles in Figure 1.

To estimate the proportion of each animal's performance that could be attributed to turning bias alone, we performed a Monte Carlo simulation. We first quantified the degree of bias for each animal by determining the number of turns of each magnitude and direction for each session. By our convention, a turn of magnitude zero represented a return to the same arm from which the animal had just emerged. A turn of +2 was an entry, via a right turn, into an arm two doors to the right of the point of emergence. A turn of -4 represented an entry into the arm directly opposite the point of emergence, via an initial left turn. Note that, with these conventions, +6 was not equivalent to -2 , since the initial turn direction was recorded independently from the succeeding arm choice.

These turn categories were then summed across sessions to produce an aggregate probability distribution for each animal. The predominant direction of turning upon leaving an arm differed among ses- sions, so the degree of asymmetry in the aggregate distribution was preserved by reversing, from left to right, those distributions with a predominance of left turns before taking the sum. The pooled distributions for each species are displayed in Figure 2. The performance of an animal that relied only on this probability distribution to determine the sequence of its choices was simulated by generating sequential, random selections from the empirical distributions. For each animal, 200 simulated sessions of 16 choices were produced. Curves of the average simulated performance of each species, based on turn bias alone, are indicated by filled circles in Figure 1.

The curves of observed and simulated performance were compared using their proportional deviation from an expectation of random, independent choices. If the task had been performed without error, the correct choice probabilities would be 1 for choices 1 through 8 and 0 for all others. The deviation from chance thus has a maximum value: the area between the curve for perfect performance, shown with open squares in Figure 1, and the random expectation, shown with open circles. We therefore characterized the performance of each animal by calculating the area between its empirical curve and the curve of random expectation and dividing by the maximum possible area. This ratio, which we term "efficiency," is mathematically equivalent to Olton and Samuelson's (1976) relative probability measure, summed across the first 16 choices. It can take any value between $100 \%$ and $-88.6 \%$, a negative value indicating per-
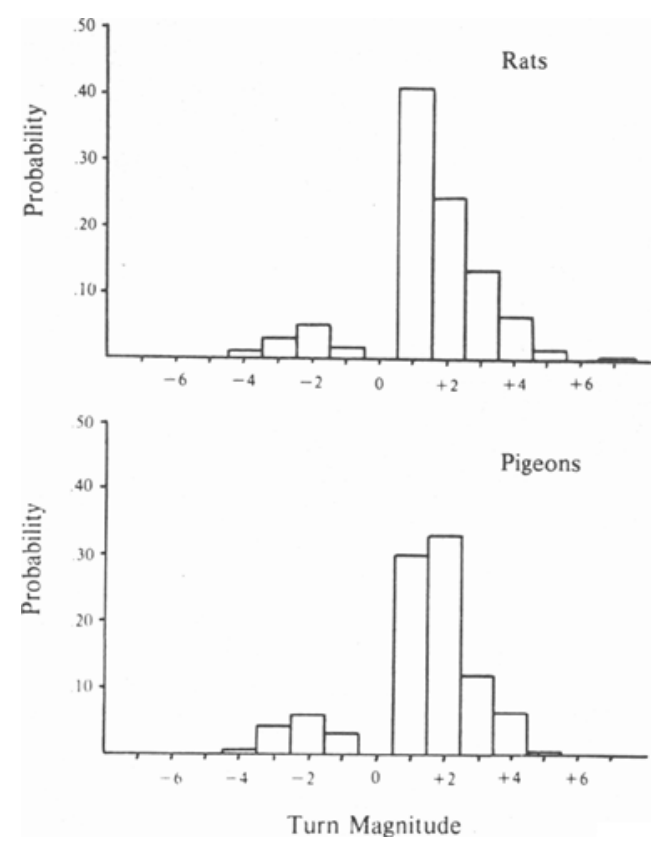

Figure 2. Probablity histogram, pooled across animals, for turn magnitude. 
formance at less than random levels, due to repeated searching of the same arm.

The efficiencies for each animal and for its Monte Carlo simulation are listed in Table 1. All treatment means were significantly greater than zero $(t>5.3$, $p<.01$ ), indicating that both observed and simulated performance were superior to random expectation. Rats were significantly more efficient than pigeons at clearing the maze $[t(14)=5.47, \mathrm{p}<.001]$. In both species, the observed performance was significantly better than that generated by the simulation model [for rats, $t(18)=7.44, p<.001$; for pigeons, $t(10)=1.84, p<.05$; one-tailed tests], implying that turning bias alone could account for only a portion of the deviation from chance.

A bias in turn magnitude and direction is the easiest alternative response strategy to describe and test. There are, unfortunately, an unlimited number of other possible strategies, incorporating different patterns and degrees of correlation among sequential arm choices, that are less easily diagnosed. The evidence for spatial-event memory is, therefore, necessarily equivocal, and inferences concerning memory capacity must be made with caution. This limitation aside, however, it is possible to obtain an estimate of the maximum memorial demand consistent with a particular level of performance using a simple memory model that assumes that the animal remembers some subset $\mathrm{m}$, termed the "memory set," of its previous arm choices. In making the next choice, it avoids the members of the memory set and selects randomly among the remaining arms.

The expected values for the correct choice probabilities in this model follow from the expression for the random expectation given previously. On a maze with $\mathrm{n}$ arms, the probability of a correct choice is 1 for all choices $<m$; for each choice $r>m$, the expectation is $[(n-m-1) /(n-m)]^{r-m-1}$. We generated a predicted probability curve for each memory set size between 0 and $n-1$ and compared the predictions to the observed functions for each animal. That value of $m$ that minimized the squared difference from the empirical curve was considered the

Table 1

Searching Efficiencies

\begin{tabular}{ccccc}
\hline & \multicolumn{2}{c}{ Rats } & \multicolumn{2}{c}{ Pigeons } \\
\cline { 2 - 5 } Animal & Observed & Simulated & Observed & Simulated \\
\hline 1 & 82.4 & 12.0 & 46.1 & 23.7 \\
2 & 95.6 & 21.0 & 36.3 & 8.8 \\
3 & 91.0 & 64.3 & 14.3 & 23.2 \\
4 & 91.2 & 14.7 & 49.5 & 20.8 \\
5 & 42.9 & 14.0 & 47.3 & 21.3 \\
6 & 91.2 & 31.5 & 25.3 & 41.6 \\
7 & 69.3 & 33.7 & & \\
8 & 95.6 & 21.6 & & \\
9 & 69.3 & 27.9 & & \\
10 & 73.7 & 30.3 & & \\
$\bar{X}$ & 80.2 & 27.1 & 36.5 & 23.2 \\
\hline
\end{tabular}

Table 2

Memory Set Size

\begin{tabular}{ccc}
\hline Animal & Rats & Pigeons \\
\hline 1 & 6 & 4 \\
2 & 7 & 2 \\
3 & 7 & 2 \\
4 & 7 & 4 \\
5 & 3 & 4 \\
6 & 7 & 3 \\
7 & 6 & \\
8 & 7 & \\
9 & 6 & \\
10 & 6 & 3.2 \\
$\bar{X}$ & 6.2 & \\
\hline
\end{tabular}

best-fit estimate of the size of the memory set. These values for each animal are listed in Table 2 . The differences among treatment means paralleled those for the efficiency measure. Again, all means were significantly greater than zero $(t>6.2, p<.001)$. The mean size of the best-fit memory set in rats was 6.2 , significantly higher than the pigeon's value of 3.2 $[t(14)=5.12, \mathrm{p}<.001]$.

The correct choice data were analyzed a third time to evaluate the possibility that the poorer performance of the pigeons was due to their not having reached an asymptotic level of performance prior to testing. The probability of a correct choice was pooled across animals within each of the 10 sessions for both species. Efficiency measures extracted from these curves were subjected to a nonparametric trend analysis (Kendall's rank correlation coefficient) to test for the occurrence of significant changes over the experimental period. There was some evidence of an improvement in performance across sessions in the rat data $[\operatorname{tau}(10)=.42, p=.054]$, but no trend was apparent in the pigeons $[\operatorname{tau}(10)=.04, \mathrm{p}>.45]$.

\section{DISCUSSION}

Rats rapidly acquired the strategy of searching each arm once, rarely repeating visits. Their performance was, therefore, consistent with the results of previous studies, in spite of the differences in the maze apparatus, such as the increase in arm length and the presence of wire-mesh barriers. Although they exhibited nonrandom turn biases, these alone were not sufficient to account for the magnitude of the effect. Our design does not eliminate other nonmemorial explanations, such as the use of odor trails, but the evidence from other studies suggests that they are not required (Olton \& Collison, 1979; Zoladek \& Roberts, 1978). Our simple memory model suggests that a capacity of about six arms would be sufficient to reproduce the rats' performance. They might well be managing with less, if one takes their response biases into account. Since half of our animals reached a performance ceiling on this task, we agree with Olton et al. (1977) that, whatever memory strategy 
rats employ in the eight-arm maze, it is by no means the limit of their abilities.

Pigeons, on the other hand, displayed only a minimal gain in performance over what could be attributed to the effects of persistent turn asymmetry. The best-fit memory set size for pigeons was about three items. Since this inferred capacity is about half of what was obtained from rats in the same apparatus, the results are in agreement with the resourcedistribution hypothesis. The estimate of a memory set of three, furthermore, is a maximum value. Given the turning bias, the birds had no need to recall the most recent arms they had visited.

Furthermore, our subjective impression, while recording the behavior of the birds, was that their turning bias disappeared after the first unrewarded choice. Olton and Samuelson (1976) have reported results from rat studies that are open to a similar interpretation. Because the turn probability distributions used as input for the simulation were derived from performance on all 16 choices, rather than merely those that preceded the first error, the simulation could have overestimated the randomness of the animals' movements in the first 8 or 9 choices and underestimated the explanatory strength of the turn bias. This systematic effect of choice number on the distribution of turn probabilities might be enough to account for the superiority of the observed performance to the simulation, at least in the pigeons. At best, therefore, the birds were using only half the memory capacity of the rats; at worst, they might not have been using spatial memory at all.

One must be cautious in interpreting negative results in studies of this kind, since it is never possible to be certain that they do not reflect a design artifact, a consequence of some feature of the apparatus that unintentionally prevents the animal from displaying its true abilities. The task appears to place roughly comparable demands on rats and pigeons, in terms of both sensory capabilities and characteristic foraging behavior, but there may be less obvious species differences that could have influenced the birds' performance. In short-term memory experiments using conditional discriminations, pigeons appear to engage in behavioral "rehearsal" (Blough, 1959; Cook, 1980). The extensive movements the maze requires appear to be incompatible with this memorial strategy. If these behaviors are essential to effective recall of recent stimuli, a poor performance on this task is inevitable. In addition, there was some suggestion that the birds tended to orient toward unsearched arms while they were at the end of an adjacent arm. The wire mesh constrained the bird from moving directly to the visible food dish, forcing it instead to walk away from the goal and back to the central platform. It seems possible that such a forced detour could have disrupted memory for the most recent arm.
Other features of the experimental design could also have contributed to the poor performance of the pigeons. It is conceivable that the difference between pigeons and rats is not one of memory capacity, but of the amount of experience required to attain a particular level of competence. If rate of learning were the critical variable, however, we might expect to see a gradual improvement in the birds' performance across the 10 sessions, and this was not the case. It is also possible that they were not sufficiently motivated to learn the task (Bitterman, 1960). The motivational level we used is fairly standard for producing reliable learning in an operant chamber, but it might have been inadequate for a design that required extensive locomotion and long intervals between arm choice and reward.

As Hailman (1976) makes clear, an ideal design for strong inference in comparative psychology requires more than a two-species comparison. Rats and pigeons differ from one another on many grounds. They are but distantly related phylogenetically, their neuroanatomy is dissimilar, and there are substantial differences in their natural history, differences other than just the characteristic distribution of their food resources. To bolster the resource-distribution argument, the radial maze procedure must ultimately be applied to at least two other species: a rodent, such as a guinea pig or vole, that customarily experiences reliable, abundant food resources and a bird that is ecologically analogous to the Norway rat, perhaps a crow or blackbird. Again, care must be taken to insure that the performance requirements of the task place comparable demands on each of the species chosen. The comparison conducted in the present experiment is a useful first step, however. It provides the best extant evidence bearing on the assumption, often made by researchers in spatial memory (Olton \& Schlosberg, 1978), that the radialarm maze taps an ability that is innate and particular to the Norway rat. At the very least, our results confirm that the rat's performance is not universal among vertebrates and suggest the usefulness of evolutionary arguments in the development of a comparative psychology of cognitive processes.

\section{REFERENCES}

Bitterman, M. E. Toward a comparative psychology of learning. American Psychologist, 1960, 15, 704-712.

Blough, D. S. Delayed matching in the pigeon. Journal of the Experimental Analysis of Behavior, 1959, 2, 151-160.

Cook, R. G. Retroactive interference in pigeon short-term memory by a reduction in ambient illumination. Journal of Experimental Psychology: Animal Behavior Processes, 1980, 6, 326-338.

GiLL, F. B., \& WoLF, L. L. Nonrandom foraging by sunbirds in a patchy environment. Ecology, 1977, 58, 1284-1296.

Goodwin, D. Pigeons and doves of the world. London: British Museum, 1967.

HaIlman, J. P. Uses of the comparative study of behavior. In 
R. B. Masterton, W. Hodos, \& H. Jerison (Ed.), Evolution, brain and behavior. Hillsdale, N.J: Erlbaum, 1976.

Hinde, R. A., \& Stevenson-Hinde, J. Constraints on learning. London: Academic Press, 1973.

KAmIL, A. C. Systematic foraging by a nectar-feeding bird, the amakihi (Loxops virens). Journal of Comparative and Physiological Psychology, 1978, 92, 388-396.

LEVI, W. M. The pigeon. Sumter, S.C: Levi, 1974.

Magni, S., Krekule, I., \& Bureš, J. Radial maze type as a determinant of the choice behavior of rats. Journal of Neuroscience Methods, 1979, 1, 343-352.

OLton, D. S. Characteristics of spatial memory. In S. H. Hulse, H. Fowler, \& W. K. Honig (Eds.), Cognitive processes in animal behavior. Hillsdale, N.J: Erlbaum, 1978.

Olton, D. S., \& Collison, C. Intramaze cues and "odor trails" fail to direct choice behavior on an elevated maze. Animal Learning \& Behavior, 1979, 7, 221-223.
Olton, D. S., Collison, C., \& Werz, M. A. Spatial memory and radial arm maze performance of rats. Learning and Motivation, 1977, 8, 289-314.

Olton, D. S., \& Samuelson, R. J. Remembrance of places passed: Spatial memory in rats. Journal of Experimental Psychology: Animal Behavior Processes, 1976, 2, 97-116.

Olton, D. S., \& Schlosberg, P. Food-searching strategies in young rats: Win-shift predominates over win-stay. Journal of Comparative and Physiological Psychology, 1978, 92, 609-618.

PARzen, E. Modern probability theory and its applications. New York: Wiley, 1960.

Zol,ADEK, L., \& Roberts, W. A. The sensory basis of spatial memory in the rat. Animal Learning \& Behavior, 1978, 6, 77-81.

(Manuscript received September 23, 1980; revision accepted for publication May 6, 1981.) 